\title{
1 Modeling of the binodal curve of ionic liquid/salt aqueous
}

\section{Systems}

3

4 Enrique Alvarez-Guerra, ${ }^{a}$ Sónia P.M. Ventura, ${ }^{\mathrm{b}}$ Manuel Alvarez-Guerra, ${ }^{*}$ a 5 João A.P. Coutinho, ${ }^{b}$ Angel Irabien ${ }^{a}$

$6 \quad{ }^{a}$ Departamento de Ingenierías Química y Biomolecular, Universidad de Cantabria. Avenida de 7 los Castros s/n, 39005 Santander, Cantabria, Spain

$8 \quad{ }^{b}$ Departamento de Química, CICECO, Universidade de Aveiro, 3810-193 Aveiro, Portugal

$9{ }^{*}$ Correspondence author: Tel.: +34 9422067 77; fax: +34 9422015 91. E-mail address: 10 alvarezgm@unican.es (M. Alvarez-Guerra).

Abstract Ionic Liquid-based Aqueous Two Phase Systems (ILATPS) are an innovative technique to separate biomolecules that combines the advantages of liquid-liquid extraction and hydrophilic ionic liquids. Most ILATPS are based on ionic liquids and conventional inorganic salts, and the phase envelope, described by the binodal curve, is usually modeled by empirical equations that are used to determine the phase compositions and assess the ionic liquid recyclability. However, these empirical equations may provide a poor extrapolation ability or low accuracy at the extreme regions of the binodal curve or suffer from problems of convergence. Therefore, the aim of this work is the analysis of the binodal curve equations, comparing the models reported in the literature to describe ILATPS and proposing alternative equations to improve accuracy or to reduce the mathematical complexity. For this purpose, a database compiling binodal experimental 
24 data of 100 ILATPS has been built, so that the analysis could make it possible to obtain representative conclusions for all these systems. Several models were developed, and different statistical criteria were used to assess the advantages and disadvantages of each one of these models for the binodal curve. The results show that, when accuracy is critical, a proposed model with just an additional parameter reduced more than $25 \%$ the residual mean squared error (RMSE) with respect to the commonly used equation, without losing the statistical significance of the parameters. For complex problems where an explicit equation in both the concentration of ionic liquid and of salt is needed, the use of an explicit model developed with 3 adjusted parameters that kept high accuracy $\left(\mathrm{R}^{2}>0.996\right.$ and RMSE $\left.<0.66\right)$ is proposed. Finally, the analysis also revealed that a fitting method based on the minimization of relative errors is recommended to increase the accuracy of the binodal curve at high salt concentrations, which is the crucial region for assessing the recyclability of the ionic liquid.

Keywords: aqueous biphasic system; binodal curve; model; accuracy; ionic liquid recyclability

\section{INTRODUCTION}

The separation and purification of biomolecules usually represents about $60-90 \%$ of the cost of the final product(s), so downstream processing determines the efficiency and viability of the biotechnological processes [1]. Among the multiple alternatives to separate biomolecules, Ionic Liquid-based Aqueous Two-Phase Systems (ILATPS) stand out for being an innovative technique that combines the advantages of liquidliquid extraction and ionic liquids [2,3]. ILATPS are powerful alternatives extracting biomolecules and have been widely used in the separation, concentration, and purification of proteins, amino-acids, antibiotics, antioxidants, alkaloids [4-6], among 
49 others [2]. They are based on ionic liquids and salts, which form two aqueous phases: an

50 ionic liquid-rich and a salt-rich phase. Many works can be found in literature in which

51 these systems are characterized in terms of the binodal curve, which also makes it

52 possible to compare the various systems with each other to derive information about the

53 mechanisms responsible for the phase separation and the design of novel ATPS.

54 Moreover, an accurate binodal curve is essential to experimentally determine the tie

55 lines by means of the gravimetric method and, in this way, the composition of the two

56 liquid phases [7-9].

57 However, rigorous models of the binodal curve for ILATPS with a theoretical support

58 are not available. In this way, the binodal curve of ILATPS is usually described by

59 means of the empirical equation proposed by Merchuk and collaborators $[2,10,11]$ :

$60 \quad[I L]=A \exp \left(B[S]^{0.5}-C[S]^{3}\right)$

61 where [IL] and [S] are the mass fractions of ionic liquid and salt expressed as percentage, respectively, and $A, B$ and $C$ are adjusted parameters. It should be noted that Merchuk's equation was originally proposed to describe conventional aqueous twophase systems based on polymers and salts. However, this equation also provides relatively high values of the $\mathrm{R}^{2}$ when modeling ILATPS, but it requires 5 parameters ( 2 fixed and 3 adjusted) to fit the experimental data and some limitations have been detected for this model. In this sense, a higher accuracy may be required for describing the extreme regions of the binodal curve (at very high ionic liquid or salt concentrations) $[7,12]$. The region of very high salt mass fractions is essential to assess the ionic liquid recyclability to the process, so the accuracy of the binodal curve in this region is particularly important [13]. In addition, Eq. 1 may cause problems of convergence when it is used in the resolution of more complex problems (recyclability experimental schemes, for example) due to the fact that it is clearly non-linear and 
74 implicit in salt concentration [14]. Therefore, the development of alternative models of

75 the binodal curve that overcome these drawbacks is particularly interesting. In the

76 literature, other empirical expressions have been proposed as alternative models to

77 enhance the accuracy [15-17]:

$78 \quad[I L]=\exp \left(a+b[S]^{0.5}+c[S]+d[S]^{2}\right)$

$79 \quad[I L]=a_{1} \exp \left(-[S] / b_{1}\right)+a_{2} \exp \left(-[S] / b_{2}\right)+c$

80 parameters:

$$
87 \ln \left(V_{213}^{*} \frac{[S]}{M_{\mathrm{S}}}+f_{213}\right)+V_{213}^{*} \frac{[I L]}{M_{\mathrm{IL}}}=0
$$

where $a, a_{1}, a_{2}, b, b_{1}, b_{2}$ and $c$ are adjusted parameters. Both equations 2 and 3 contain a higher number of adjusted parameters (4 and 5, respectively) than Merchuk's equation. Another approach reported in previous works [15,17-19] implies a binodal curve model based on statistical geometry methods, developed by Guan et al. [20] for aqueous polymer-polymer systems. This binodal equation has a theoretical support by means of the concept of effective excluded volume (EEV) and contains only two adjusted

where $V_{213}^{*}$ is the scaled EEV of salt; $f_{213}$ is the volume fraction of unfilled effective available volume after tight packing of the salt molecules into the ionic liquid molecules network in ionic liquid aqueous solutions; and $M_{\mathrm{S}}$ and $M_{\mathrm{IL}}$ are the molecular masses of the salt and the ionic liquid, respectively. It should be highlighted that only this binodal curve model has some theoretical foundation, in contrast with the remaining models, which are purely empirical. Few studies have carried out a comparison among models for the binodal curve $[15,17,21]$. Nevertheless, these analyses have been done using a reduced number of systems (lower than 10 in all the cases) and very simple statistical criteria, such as the standard deviation and/or the $\mathrm{R}^{2}$ coefficient. As a result, the 
97 conclusions derived from these works with respect to the selection of the binodal curve

model cannot be easily extrapolated to the hundreds of ILATPS described in literature.

In this way, the aim of this work is the analysis of the binodal curve equation to describe ILATPS based on ionic liquids and salts, comparing the previous models and proposing either alternative equations which may improve its accuracy or simpler the mathematical models that keep successful performances. For this purpose, a database with the binodal data of 100 ILATPS was built and subsequently analyzed so that the conclusions obtained are representative for all these systems. Furthermore, different statistical criteria have been used in order to discuss in detail the advantages and disadvantages of each binodal equation.

\section{METHODS}

\subsection{Methodology}

The methodology followed in this study is graphically summarized in Fig. 1.

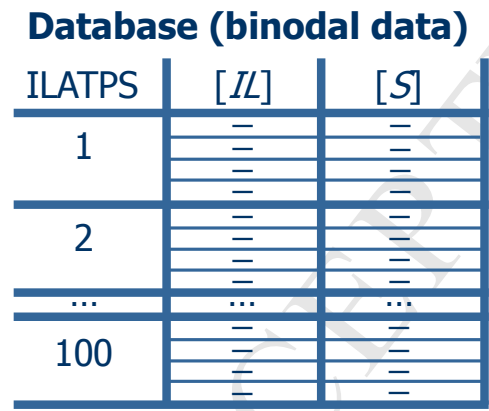

30 different ILs and 9 different (inorganic and organic) salts

\begin{tabular}{c|c|c|c} 
& Mean R2 & Mean F & $\ldots$ \\
\hline Model 1 & - & - & $\ldots$ \\
\hline Model 2 & - & - & $\ldots$ \\
\hline$\ldots$ & $\ldots$ & $\ldots$ & $\ldots$
\end{tabular}

Statistical criteria for model discrimination and selection

\section{Data fitting to binodal curve models}
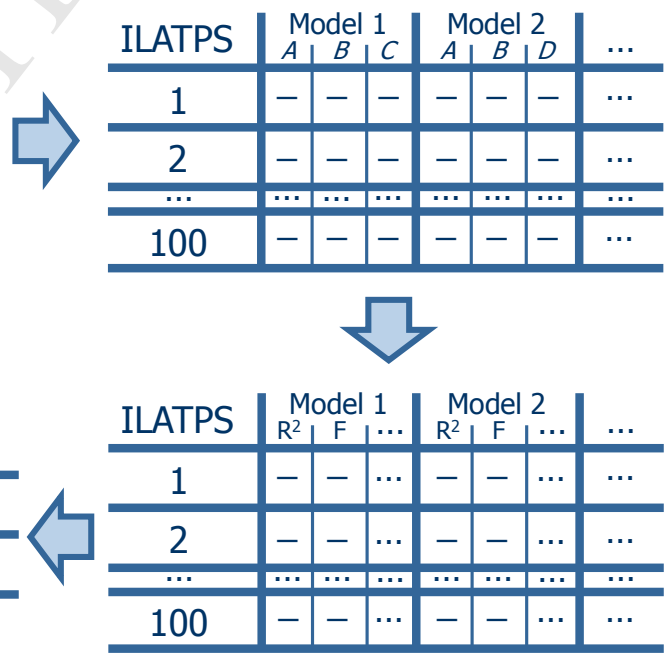

Statistical criteria related to each ILATPS and model

Fig. 1-Scheme of the methodology followed in this work. 
114 Binodal data from 100 ILATPS systems were compiled from the literature [7,22-30] in

115 order to assure that the conclusions derived from the study are representative of these

116 types of systems. The database created included 100 ILATPS systems, which involved

11730 different ILs and 9 different (inorganic and organic) salts. The binodal curves of

118 these ILATPS were determined at room temperature, as liquid-liquid extraction with

119 these systems is usually carried out at this temperature. In addition, it is expected that

120 the conclusions derived in this work from the analysis of the binodal curve of ILATPS

121 at room temperature can also be applied to other temperatures, as the linear dependency

122 of the adjusted parameters of Merchuk's equation with respect to temperature suggests.

123 With respect to the influence of the temperature on ILATPS, the biphasic region 124 decreases with the increase in this variable, which implies that the higher the temperature, the higher the salt and ionic liquid concentrations required for phase separation. However, the intensity of the temperature effect on the phase diagrams depends on the inorganic salt employed [2]. The complete dataset is included in Table S1 as Supplementary Material. For each ILATPS, the binodal data were fitted to each model that was considered in the study, obtaining the values of the adjusted parameters and the statistical criteria that will be described in section 2.2. Finally, for the discrimination and selection of the models, the means of the statistical criteria were calculated, and these means, for the 100 ILATPS, are the values that will be reported in section 3 "Results and discussion".

\subsection{Statistical criteria for the discrimination and model selection}

The discrimination and selection of the binodal curve models has been carried by means of different statistical criteria that consider the accuracy, the significance of the parameters or the number of adjusted parameters [31]: 
139 - Coefficient of determination $\left(\mathrm{R}^{2}\right)$, which indicates the proportionate amount of

140 variation in the response explained by the independent variable.

141 - The wideness of the confidence interval, which is a measure of the significance of the

142 parameters; if a parameter is significant, it should not contain the zero value.

143 - Fischer's F value $(\mathrm{F})$, which is based on a null hypothesis that advocates for the 144 adequacy of the model to the observed values of the measured variable.

145 - The residual mean squared error (RMSE), which is often considered a measure of the 146 difference of the predicted values of the variable and the experimental observations.

147 - The Akaike information criterion (AIC), which gives information of the goodness of 148 the fit while penalizing model overfitting by increasing the number of parameters of the 149 model.

150 In general, the quality of each model to describe the experimental data increases with the value of $\mathrm{R}^{2}$ and $\mathrm{F}$, and as the wideness of the confidence interval, the RMSE and the AIC decrease. As previously explained, each model will be assessed by the mean value of these statistical criteria obtained for each of the ILATPS included in the database. However, in the case of the wideness of the confidence interval, as this criterion is determined as the mean of relative wideness (with respect to the value of each parameter) of all the parameters for each ILATPS, to avoid the interference of the extreme values, each model is characterized by the median wideness of the 100 ILATPS instead of by the mean value.

\section{RESULTS AND DISCUSSION}

\subsection{Assessment of the exponents of the Merchuk's equation}

As previously stated, Merchuk's equation is widely applied to fit the binodal curve of 
164 [10]. In this sense, it should be noticed that this equation contains two constant

165 parameters that correspond to both exponents (see Eq. 1), meaning that the values used

166 for the polymer-salt ATPS (0.5 and 3.0) may not be the most suitable ones to model

167 ILATPS. Therefore, alternative values for the exponents of Merchuk's equation were

168 tested in order to assess if the fitting of the binodal data can be improved. To carry out

169 this assessment, the binodal data of the 100 ILATPS systems of the database were fitted

170 using different combinations of values of the Merchuk's equation exponents. The

171 results of the mean $\mathrm{R}^{2}$ obtained for each pair of values of the exponents are summarized

172 in Table 1.

174 Table 1-Mean $\mathrm{R}^{2}$ obtained in the fitting of the ILATPS of the database of this study 175 using different values of exponents $\mathrm{D}$ and $\mathrm{E}$ of Merchuk's equation: $[\mathrm{IL}]=\mathrm{A} \exp (\mathrm{B}$ $\left.176 \quad[\mathrm{~S}]^{\mathrm{D}}-\mathrm{C}[\mathrm{S}]^{\mathrm{E}}\right)$

\begin{tabular}{|l|lcc|}
\hline \multirow{2}{*}{$\boldsymbol{E}$} & \multicolumn{1}{|l}{} & \\
\cline { 2 - 4 } & $\mathbf{0 . 4}$ & $\mathbf{0 . 5}$ & $\mathbf{0 . 6}$ \\
\hline $\mathbf{2 . 5}$ & 0.99812 & 0.99813 & 0.99799 \\
$\mathbf{3 . 0}$ & 0.99794 & $\mathbf{0 . 9 9 8 1 5}$ & 0.99812 \\
$\mathbf{3 . 5}$ & 0.99747 & 0.99794 & 0.99808 \\
\hline
\end{tabular}

177

178 The fitting of these mean $\mathrm{R}^{2}$ values to a quadratic polynomial expression (Fig. 2) shows

179 how they vary depending on the values of the exponents D and E used. The maximum

180 value of mean $\mathrm{R}^{2}$ for the 100 ILAPTS was obtained when exponents were $\mathrm{D}=0.506$ and

$181 \mathrm{E}=2.74$. Consequently, the binodal data of these systems were fitted using these values

182 of the exponents, obtaining that this maximum mean $\mathrm{R}^{2}$ was equal to 0.99817 , which

183 resulted to be very similar to the value of 0.99815 obtained with the original Merchuk's

184 equation (i.e. $\mathrm{D}=0.5, \mathrm{E}=3.0$, Table 1 ). As can be seen, there is a region of combinations

185 (D, E) in which almost the same mean $\mathrm{R}^{2}$ value is obtained (higher than 0.9981 ) and

186 that contains the original values of Merchuk's equation. Since the studies in the 
187 literature that model the binodal curve of ILAPTS with Merchuk's equation routinely

188 use 0.5 and 3.0 as values for the exponents, the very small increase in $\mathrm{R}^{2}$ obtained does

189 not justify to propose the change of these exponents. Although the values of the

190 exponents $(0.5,3.0)$ are empirical and were developed for conventional polymer-salt

191 ATPS, the results of our study reveal that they belong to the region of values $(\mathrm{D}, \mathrm{E})$ that

192 describe the binodal curve of ILATPS with the highest accuracy when this equation is 193 used.

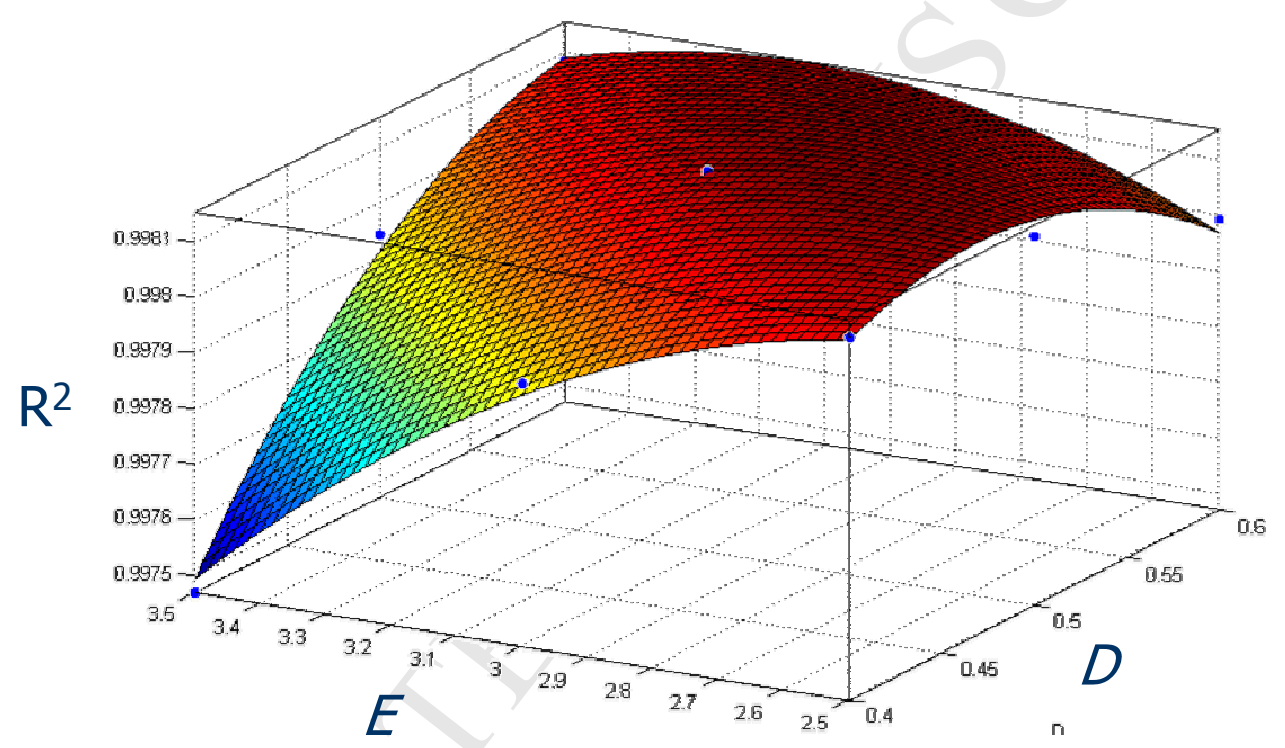

Fig. 2-Fitting of the mean $\mathrm{R}^{2}$ to the quadratic polynomial function depending on the values of Merchuk's equation exponents.

\subsection{Development and assessment of alternative binodal curve models for ILATPS}

Different models were developed and their performance when describing the binodal the Merchuk's equation tested, considering models with a different number of total parameters and adjusted parameters, and also models explicit in both variables (i.e. [IL], concentration of IL and [S], concentration of salt) or not. The mean values of statistical criteria $\mathrm{R}^{2}, \mathrm{~F}, \mathrm{RSME}$ and AIC obtained when each of these models was used for the 
ILATPS of the database are reported in Table 3, together with the confidence intervals,

207 for which the median values are reported due to the higher robustness to extreme values

208 that can be obtained in some ILATPS, as previously mentioned.

209

210 Table 2-Models assessed to describe the binodal curve of ILATPS.

\begin{tabular}{|llccc|}
\hline $\begin{array}{c}\text { Model } \\
\text { No. }\end{array}$ & Model & $\begin{array}{c}\text { No. of } \\
\text { parameters }\end{array}$ & $\begin{array}{c}\text { No. of adjusted } \\
\text { parameters }\end{array}$ & $\begin{array}{c}\text { Explicit in } \\
\text { the two } \\
\text { variables }\end{array}$ \\
\hline 1 & {$[I L]=A \cdot \exp \left(B[S]^{D}-C[S]^{3}\right)$} & 5 & 4 & NO \\
2 & {$[I L]=A \cdot \exp \left(B[S]^{0.5}-C[S]^{E}\right)$} & 5 & 4 & NO \\
3 & {$[I L]=A \cdot \exp \left(B[S]^{0.5}-C[S]^{3}\right)$} & 5 & 3 & NO \\
4 & {$[I L]=A \cdot \exp \left(B[S]^{D}\right)$} & 3 & 3 & YES \\
5 & {$[I L]=A \cdot \exp (B[S])$} & 2 & 2 & YES \\
6 & {$[I L]=\exp \left(a+b[S]^{0.5}+c[S]+d[S]^{2}\right)$} & 6 & 4 & NO \\
7 & $\ln \left(V_{213}^{*} \frac{[S]}{M_{S}}+f_{213}\right)+V_{213}^{*} \frac{[I L]}{M_{I L}}=0$ & 4 & 2 & YES \\
\hline
\end{tabular}

211

212 Table 3-Summary of statistical criteria for the assessment of the models developed.

213 Model numbers correspond to those listed in Table 2.

\begin{tabular}{|cccccc|}
\hline $\begin{array}{c}\text { Model } \\
\text { No. }\end{array}$ & Mean R $^{2}$ & $\begin{array}{c}\text { Median confidence } \\
\text { interval }\end{array}$ & Mean F & Mean RSME & Mean AIC \\
\hline 1 & 0.9991 & $27.50 \%$ & $2.088 \cdot 10^{5}$ & 0.3264 & -137.4 \\
2 & 0.9989 & $47.86 \%$ & $1.704 \cdot 10^{5}$ & 0.3585 & -128.7 \\
3 & 0.9981 & $5.728 \%$ & $1.556 \cdot 10^{5}$ & 0.4469 & -108.4 \\
4 & 0.9964 & $11.67 \%$ & $5.559 \cdot 10^{4}$ & 0.6579 & -55.07 \\
5 & 0.9900 & $2.694 \%$ & $3.406 \cdot 10^{4}$ & 1.025 & -12.08 \\
6 & 0.9992 & $22.01 \%$ & $1.990 \cdot 10^{5}$ & 0.3188 & -137.4 \\
7 & 0.9686 & $13.73 \%$ & $2.035 \cdot 10^{4}$ & 1.646 & 41.56 \\
\hline
\end{tabular}

215 As shown in Table 2, Models 1 and 2 included an additional adjusted parameter 216 compared to the original Merchuk's equation (Model 3). Table 3 shows that both

217 Models 1 and 2 gave very similar results, enhancing the accuracy with respect to the 218 performance of the Merchuk's equation (e.g. mean RSME is reduced 27\% in Model 1 and $20 \%$ in Model 2 with respect to Model 3). Moreover, despite the lower median 
confidence interval obtained with Model 3, which can be explained considering that it contains only 3 adjustable parameters instead of 4 , in most of the ILATPS assessed, the values of the parameters of model 2 and, especially, of model 1 were statistically significant, so the inclusion of the fourth parameter is advisable, which is also proved by the AIC values. However, it should be mentioned that the 5-adjusted parameter equation (not included in Tables 2 and 3) leads to overfitting, since most of the parameters were not statistically significant. In addition, even though Models 1 and 2 only differ in the exponent that is adjusted, Model 1 clearly describes better the binodal curve, so the use of Model 2 is discarded. It is also important to note that the mathematical complexity of these models is identical, since all the parameters (fixed and adjusted) are constant once their values have been obtained. Therefore, the use of Model 1 is recommended when the maximum accuracy of the binodal curve is required. All the previous models (1-3) are implicit in the salt concentration. For this reason, other models that can be explicit in the two variables ([IL] and [S]) were also developed, because, as already mentioned, reducing the mathematical complexity of the equation for the binodal curve can be important when it is used in complex modeling problems to make convergence easier. In this sense, Model 4 is outstanding, since it contains 3 adjustable parameters and it is explicit in both variables (Table 2), providing relatively high accuracy, with $\mathrm{R}^{2}>0.996$ and RMSE $<0.66$ (Table 3). Decreasing the number of adjusted parameters to 2 (model 5, Table 2), the accuracy of the fitting is considerably reduced, although maintaining an acceptable mean value of $\mathrm{R}^{2}=0.990$. More interestingly and as shown in Table 3, Model 5 shows the narrowest error range of all models, which implies that the significance is increased. Nevertheless, in the other cases, when explicit models in the two variables are more suitable, Model 4 is 
244 recommended due to its higher accuracy, which is not very different from that obtained

245 with the original Merchuk's equation (Model 3).

246 Other models, previously reported in literature and following distinct approaches from

247 the Merchuk's equation, were also analyzed in this work. Model 6 is another empirical

248 model with 6 parameters (considering both fixed and adjusted), while Model 7 is the

249 theoretical model originally developed for aqueous polymer-polymer systems but that

250 has been proposed to be used also for ILATPS [15,17-19]. It should be mentioned that

251 tests with the model of Eq. 3 were also carried out, although the results are not shown

252 because it suffered from computational problems and, in the cases where it was possible

253 to obtain adjusted parameters, most of them were not significant.

254 It can be seen in Table 3 that Model 6 leads to a goodness of fit almost identical to that provided by Model 1, but it contains an additional fixed parameter. Furthermore, considering that Merchuk's equation is more widely used than Model 6, the replacement of Model 1 by Model 6 is not recommended. On the other hand, Model 7 clearly shows the poorest results among all the models analyzed. Even though Models 5 and 7 contain the same number of adjustable parameters and Model 7 has two additional fixed parameters, Model 5 clearly describes the binodal data of ILATPS more accurately. In addition, it should be noted that the parameter significance of models 3 and 4 is higher than the significance of model 7 , since the latter leads to a wider median confidence interval even though it implies one adjusted parameter less. This confirms that the use of model 7 is not advisable to describe the binodal curve of ILATPS. Therefore, these results reveal that the approaches such as Model 7 based on the calculation of the effective excluded volume (EEV), which were developed for aqueous polymer-polymer systems, cannot describe adequately the binodal curve of ILATPS because the 
268 assumptions involved in this theory [15,17-19] cannot be extended to ionic

269 liquid/salt/water systems.

270 Finally, in order to exemplify how each model describes the binodal data and

271 extrapolates the binodal curve to a wider range of compositions, Figure 3 shows the

272 experimental binodal data and the binodal curve simulated by each model of the

273 ILATPS formed by $\left[\mathrm{P}_{i(444) 1}\right][\mathrm{Tos}]$ as ionic liquid and $\mathrm{K}_{2} \mathrm{HPO}_{4} / \mathrm{KH}_{2} \mathrm{PO}_{4}$ as the salt

274 component (system number 58, see Supplementary material). As can be seen, Models 1-

2756 lead to a similar fitting of the binodal data, which is in accordance with the relatively

276 high accuracy achieved by all of them $\left(\mathrm{R}^{2} \geq 0.99\right.$, AIC $\left.<0 \ldots\right)$. However, it is clear that

277 Model 7 cannot describe the trend of the binodal data, which reinforces the idea that this

278 model is not suitable for ILATPS. Regarding the values of the parameters, clear trends

279 have not been detected, although this work is not focused on predictive purposes.

280

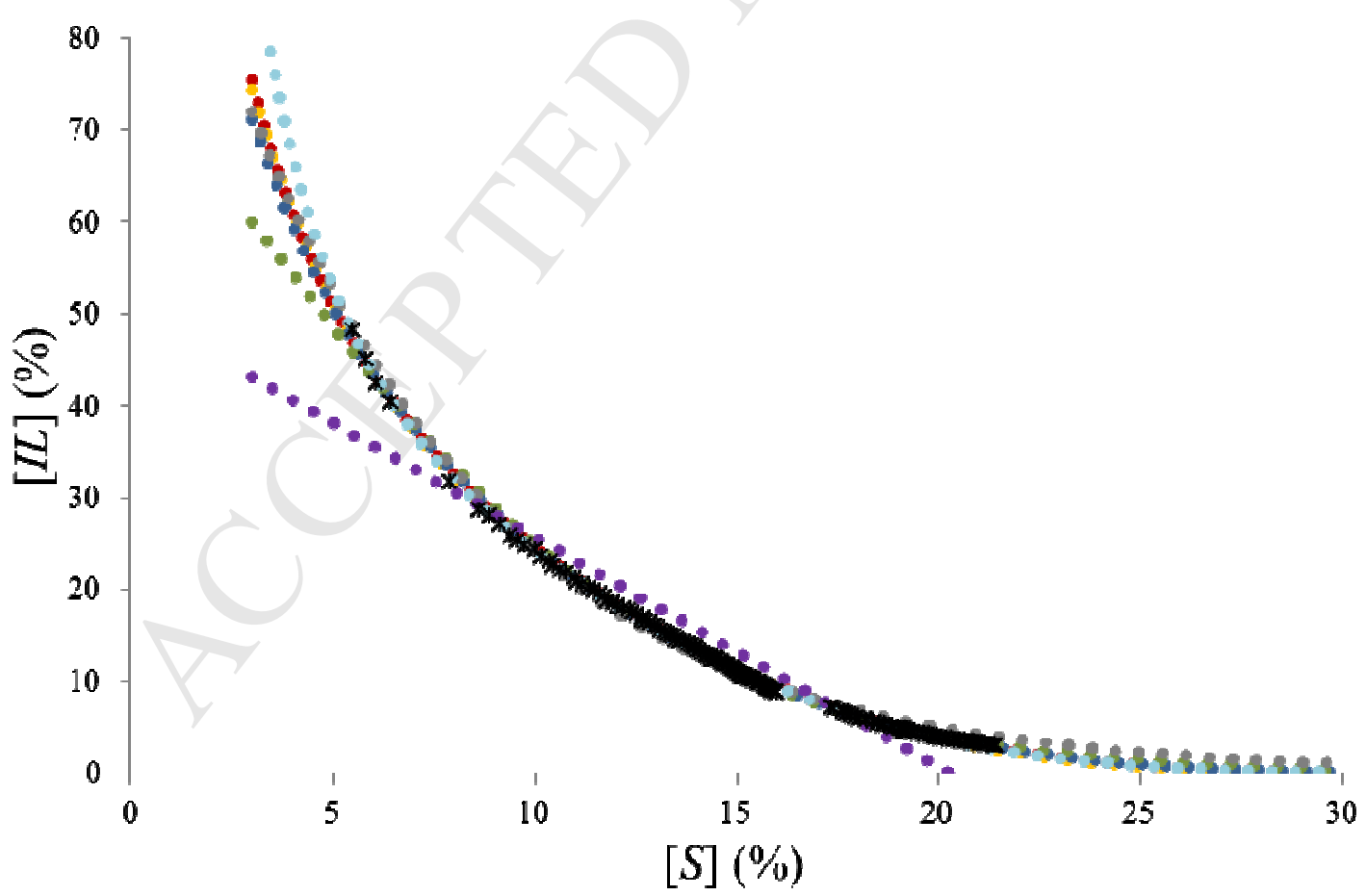

281

282 Figure 3-Binodal data of the ILTAPS formed by $\left[\mathrm{P}_{i(444) 1}\right][\mathrm{Tos}]$ as ionic liquid and $283 \mathrm{~K}_{2} \mathrm{HPO}_{4} / \mathrm{KH}_{2} \mathrm{PO}_{4}$ as the salt component (system number 58, see Supplementary 
284 material). Notation: experimental data $(\boldsymbol{*})$; simulated data by: Model $1(\bullet)$, Model 2

$285(\bullet)$, Model $3(\bullet)$, Model $4(\bullet)$, Model $5(\bullet)$, Model $6(\bullet)$ and Model $7(\bullet)$.

286

287

\subsection{Improving the accuracy at high salt mass fractions: alternative fitting method}

288

For the analysis of the recyclability of the IL in separation processes based on ILATPS,

the IL present in the salt-rich phase is critical since it may constitute the losses of the process. Therefore, the correct assessment of the IL concentration in this phase is critical, which corresponds to the region of the binodal curve at high salt concentrations imply high relative errors when determining the mentioned concentration of IL.

As usual, in the models already discussed in previous analyses, the method of least squares based on the minimization of the sum of absolute errors, $\mathrm{S}$, was used for fitting the data: Min $S=\sum_{i=1}^{n}\left(y_{i}-\hat{y}_{i}\right)^{2}$, where $y_{i}$ and $\hat{y}_{i}$ are the observed and fitted response values, respectively. In this case, the observed values correspond to the concentration of ionic liquid for a given salt concentration that defines the biphasic region in the phase diagram. Nevertheless, in this section, with the aim of improving the performance of the models at high salt mass fractions, the results obtained using an alternative method for fitting the binodal curve based on minimizing the sum of the relative errors (instead of

302 the sum of the absolute errors) is presented: Min $S=\sum_{i=1}^{n} \frac{\left|y_{i}-\hat{y}_{i}\right|}{y_{i}}$. It should be noted that the relatives errors will always be positive, since, in this case, $y_{i}$ is the experimental mass fraction of ionic liquid for a given salt concentration $i$, which is an intrinsically positive variable. 
Table 4-Comparison of the results using two alternative fitting methods: method of least squares based on minimization of absolute errors (called "least squares" in the table); and fitting method based on minimization of relative errors ("relative error"). Model numbers correspond to those listed in Table 2.

\begin{tabular}{|c|c|c|c|c|c|c|}
\hline \multirow{3}{*}{$\begin{array}{l}\text { Model } \\
\text { No. }\end{array}$} & \multicolumn{4}{|c|}{ Mean relative error in absolute value $(\%)$} & \multirow{2}{*}{\multicolumn{2}{|c|}{ Mean RSME }} \\
\hline & \multicolumn{2}{|c|}{ All binodal curve } & \multicolumn{2}{|c|}{$\begin{array}{c}5 \text { points with the lowest } \\
\text { [IL] }\end{array}$} & & \\
\hline & $\begin{array}{c}\text { Least } \\
\text { squares }\end{array}$ & $\begin{array}{l}\text { Relative } \\
\text { error }\end{array}$ & Least squares & Relative error & $\begin{array}{c}\text { Least } \\
\text { squares }\end{array}$ & $\begin{array}{c}\text { Relative } \\
\text { error }\end{array}$ \\
\hline 1 & 1.055 & 0.8983 & 2.334 & 1.453 & 0.3264 & 0.3775 \\
\hline 3 & 1.498 & 1.135 & 3.724 & 1.964 & 0.4469 & 0.6254 \\
\hline 4 & 2.633 & 1.726 & 7.000 & 2.946 & 0.6579 & 0.9975 \\
\hline 5 & 4.530 & 3.414 & 11.96 & 6.824 & 1.025 & 1.676 \\
\hline
\end{tabular}

The comparison of the results obtained using different models that were fitted with the two methods is reported in Table 4, in which the mean relative errors in absolute value have been calculated according to Equation 5:

Mean relative error in absolute value $(\%)=100 \frac{\sum_{j=1}^{n} \frac{\sum_{i=1}^{n_{j}} \frac{\left|y_{i, j}-\hat{y}_{i, j}\right|}{y_{i, j}}}{n_{j}}}{n}$

where $j$ denotes the different ILATPS included in the database and $i$ represents each binodal data of the ILTAPS $j$, so $n_{j}$ is the available number of experimental points of the binodal curve of $j$, and $n$ is the number of ILAPTS (in this particular study $n=100$ ). The models chosen for comparison are those identified as the most suitable ones to model the binodal curve, including both non explicit (Models 1 and 3) and explicit (Models 4 and 5). The results summarized in Table 4 show that the fitting methods based on the minimization of the relative errors reduced the mean relative errors between $15 \%$ (in Model 1) and $34 \%$ (in Model 4) with respect to the least squares method, keeping the mean RSME in relatively low values, which were lower than 1 for Models 1, 3 and 4.

It is particularly interesting to focus on analyzing the performance of the models with both fitting methods when dealing with the part of the binodal curve with the lowest 
328 concentration of IL, since this is the most important region of the curve to assess the

329 recyclability of the IL. As can be seen in Table 4, for the 5 points of the binodal curve 330 with the lowest concentration of IL, the models developed using the fitting method

331 based on relative errors increased the reduction of the error to the range between 38 and

$33258 \%$ compared to the corresponding models fitted with least squares. This behavior can

333 be explained considering that the least squares method tends to minimize absolute errors, whereas the alternative fitting method proposed is based on the minimization of relative errors. For this reason, assuming that in both cases the corresponding fitting errors are kept constant along the binodal curve, even high relative errors (15\%) may correspond to considerably lower absolute errors than a given low absolute error at low values of the function (i.e., the region at high salt concentration), as graphically exemplified in Fig. 4. This figure shows two generic independent and dependent variables (X and $\mathrm{Y}$, respectively), and demonstrates how the minimization of relative errors reduces considerably the absolute error in the region of low values of $\mathrm{Y}$,

342 increasing the error at high $\mathrm{Y}$. In the case of binodal curves, the region of low $\mathrm{Y}$ 343 correspond to high salt mass fractions, i.e. the zone of the binodal curve identified as 344 crucial when looking for the ionic liquid recyclability. In this way, this alternative 345 fitting method clearly improves the accuracy of the models in this important region of 346 the binodal curve but without suffering from excessive errors in others, since the 347 absolute errors measured by means of RSME remain relatively low. 

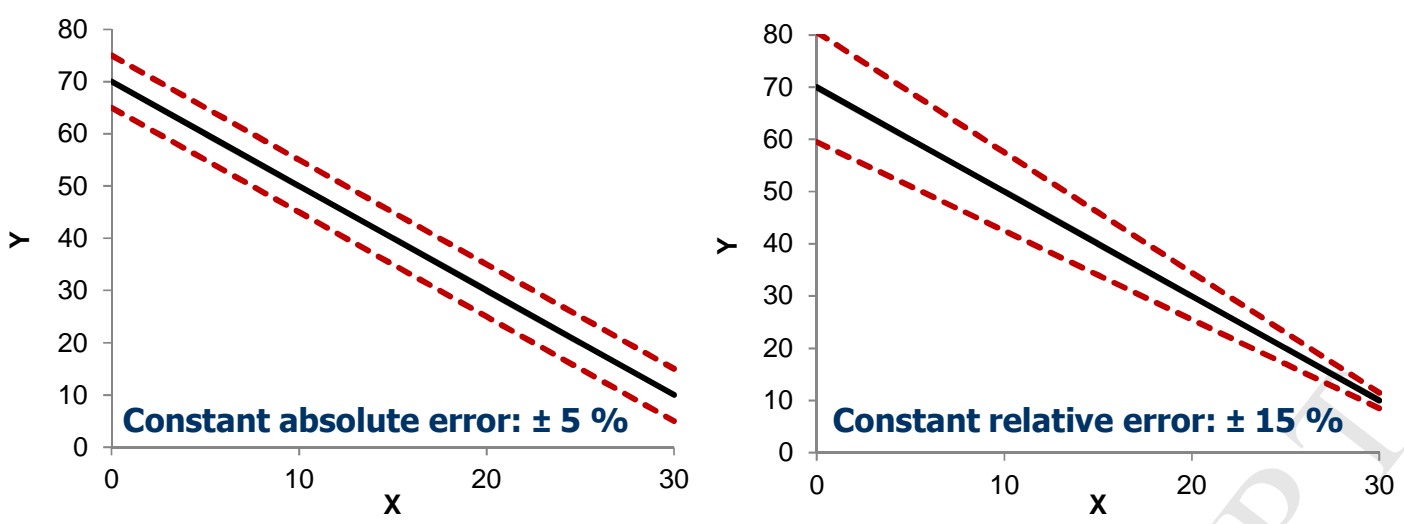

349

Fig. 4-Distribution of the fitting error at constant absolute (left) or relative (right) errors.

Therefore, these results reveal that the development of the models with a fitting method that minimizes the relative errors instead of the absolute errors allowed enhancing the accuracy of the fitting of the binodal curve of ILATPS in the most important region for carrying out the ionic liquid recyclability analyses.

\section{CONCLUSIONS}

This work provides a critical assessment of the models of binodal curves of ILATPS, and particularly, of the equation proposed by Merchuk and collaborators [10], since it is the most widely applied. The results of this study confirm that even though the empirical values of the exponents of Merchuk's equation (0.5 and 3.0) were developed for polymer-salt ATPS, they are also valid for ILATPS, fitting the binodal curve with the highest accuracy.

364 Alternative models have been proposed in this work to replace this equation with the aim of increasing the accuracy or reducing the mathematical complexity, depending on the requirements of each specific application. In this way, when accuracy is critical and

367 the binodal curve equation is not involved in complex models (i.e. convergence problems are not expected), the proposed equation to describe this equilibrium curve 
369 turns the first exponent of the Merchuk's equation into an adjusted parameter, $D$ : $[I L]=A \cdot \exp \left(B[S]^{D}-C[S]^{3}\right)$

371 However, the most complex models may suffer from convergence problems (e.g. large

372 non-linear optimization problems), so in these cases the binodal curve of ILATPS 373 should be explicit in the two variables so that iterative procedures are not required to 374 solve this clearly non-linear equation. For this purpose, the use of the following model 375 that contains 3 adjustable parameters and it is explicit in both the concentration of IL 376 and the concentration of salt, keeping relatively high accuracy $\left(\mathrm{R}^{2}>0.996\right.$ and $\mathrm{RMSE}<$ 377 0.66), is proposed: $[I L]=A \cdot \exp \left(B[S]^{D}\right)$.

378 Finally, a detailed study is carried out for the binodal curve at high salt concentrations, 379 due to the importance of this region for the recovery of the ionic liquid used in the 380 processes based on ILATPS. In this sense, the fitting method based on the minimization of relative errors is recommended to increase significantly the accuracy of the binodal curve in this crucial region for assessing the recyclability of the IL.

\section{Acknowledgements}

This work was developed in the scope of the project CICECO-Aveiro Institute of Materials (Ref. FCT UID/CTM/50011/2013), financed by national funds through the FCT/MEC and co-financed by FEDER under the PT2020 Partnership Agreement. The authors also acknowledge FCT for the Post-doctoral grant SFRH/BPD/79263/2011 of S.P.M. Ventura. 
394 [1] C.M.S.S. Neves, S.P.M. Ventura, M.G. Freire, I.M. Marrucho, J.A.P. Coutinho, 395 Evaluation of Cation Influence on the Formation and Extraction Capability of 396 Ionic-Liquid-Based Aqueous Biphasic Systems, J. Phys. Chem. B, 113 (2009) 5194-5199.

[2] M.G. Freire, A.F.M. Cláudio, J.M.M. Araújo, J.A.P. Coutinho, I.M. Marrucho, J.N.C. Lopes, L.P.N. Rebelo, Aqueous biphasic systems: a boost brought about by using ionic liquids, Chem. Soc. Rev., 41 (2012) 4966-4995.

[3] J.F.B. Pereira, S.P.M. Ventura, F.A. E Silva, S. Shahriari, M.G. Freire, J.A.P. Coutinho, Aqueous biphasic systems composed of ionic liquids and polymers: a platform for the purification of biomolecules, Sep. Purif. Technol. 113 (2013) 8389.

[4] E. Alvarez-Guerra, A. Irabien, Separation of Proteins by Ionic Liquid-Based Three-Phase Partitioning, in: A. Perez de los Rios, F.J. Hernandez-Fernandez (Eds.), Ionic Liquids in Separation Technology, Elsevier, Amsterdam, 2014, pp. 207-234.

[5] M. Domínguez-Pérez, L.I.N. Tomé, M.G. Freire, I.M. Marrucho, O. Cabeza, J.A.P. Coutinho, (Extraction of biomolecules using) aqueous biphasic systems formed by ionic liquids and aminoacids, Sep. Purif. Technol. 72 (2010) 85-91.

[6] S.P.M. Ventura, R.L.F. de Barros, J. M. de Pinho Barbosa, C.M.F. Soares, A.S. Lima, J.A.P. Coutinho, Production and Purification of an Extracellular Lipolytic Enzyme using Ionic Liquid-based Aqueous Two-phase Systems, Green Chem. 14 (2012) 734-740.

[7] M.V. Quental, H. Passos, K.A. Kurnia, J.A.P. Coutinho, M.G. Freire, Aqueous Biphasic Systems Composed of Ionic Liquids and Acetate-Based Salts: Phase Diagrams, Densities, and Viscosities, J. Chem. Eng. Data 60 (2015) 1674-1682. 
419 [8] C.F.C. Marques, T. Mourão, C.M.S.S. Neves, A.S. Lima, I. Boal-Palheiros, J.A.P.

420 Coutinho, M.G. Freire, Aqueous Biphasic Systems Composed of Ionic Liquids

421 and Sodium Carbonate as Enhanced Routes for the Extraction of Tetracycline,

422 Biotechnol. Prog., 29 (2013) 645-645.

423 [9] S. Shahriari, C.M.S.S. Neves, M.G. Freire, J.A.P. Coutinho, Role of the 424 Hofmeister Series in the Formation of Ionic-Liquid-Based Aqueous Biphasic 425 Systems, J. Phys. Chem. B, 116 (2012) 7252-7258.

426 [10] J.C. Merchuk, B.A. Andrews, J.A. Asenjo, Aqueous two-phase systems for protein separation studies on phase inversion, J. Chromatogr. B 711 (1998) 285-

428

429

430

431

432

433

434

435

436

437

438

439

440

441

442

443 293.

[11] C.M.S.S. Neves, M.G. Freire, J.A.P. Coutinho, Improved recovery of ionic liquids from contaminated aqueous streams using aluminium-based salts, RSC Adv. 2 (2012) 10882-10890.

[12] E. Alvarez-Guerra, S.P.M. Ventura, J.A.P. Coutinho, A. Irabien, Ionic Liquidbased three phase partitioning (ILTPP) systems: Ionic liquid recovery and recycling, Fluid Phase Equilibr. 371 (2014) 67-74.

[13] E. Alvarez-Guerra, S.P.M. Ventura, J.A.P. Coutinho, A. Irabien, Ionic Liquid Recovery Alternatives in Ionic Liquid-Based Three Phase Partitioning (ILTPP), AIChE J. 60 (2014) 3577-3586.

[14] E. Alvarez-Guerra, A. Irabien, Optimization of ionic liquid recycling in Ionic Liquid-based Three Phase Partitioning processes, in: K.V. Gernaey, J.K. Huusom, R. Gani (Eds.), Computer-Aided Chemical Engineering, 37, Elsevier, Amsterdam, 2015, pp. 1475-1480.

[15] Y. Li, M. Zhang, J. Wu, J. Shi, C. Shen, Liquid-liquid equilibria of ionic liquid Nbutylpyridinium tetrafluoroborate and disodium hydrogen phosphate/sodium 
chloride/sodium sulfate/ammonium sulfate aqueous two-phase systems at $T=298.15$ K: Experiment and correlation, Fluid Phase Equilibr. 378 (2014) 44-50.

446

447

[16] W. Zhang, G. Zhang, J. Han, Y. Yan, B. Chen, C. Sheng, Y. Liu, Phase equilibrium and chloroamphenicol partitioning in aqueous two-phase system composed of 1-hydroxylhexyl-3-methylimidazolium chloride-salt, J. Mol. Liq. 193 (2014) 226-231.

[17] Y. Li, L. Yang, X. Zhao, W. Guan, Liquid-liquid equilibria of ionic liquid Nethylpyridinium tetrafluoroborate + trisodium citrate/ammonium citrate tribasic/sodium succinate/sodium tartrate aqueous two-phase systems at $298.15 \mathrm{~K}$, Thermochim. Acta 550 (2012) 5-12.

[18] I. Regupathi, S.L. Monteiro, 1-Hexyl-3-Methylimidazolium Chloride - Potassium Carbonate Aqueous Two Phase System: Equilibrium Characteristics and BSA Partitioning Behavior, J. Disper. Sci. Technol. 35 (2014) 418-427.

[19] J. Han, C. Yu, Y. Wang, X. Xie, Y. Yan, G. Yin, W. Guan, Liquid-liquid equilibria of ionic liquid 1-butyl-3-methylimidazolium tetrafluoroborate and sodium citrate/tartrate/acetate aqueous two-phase systems at $298.15 \mathrm{~K}$ : Experiment and correlation, Fluid Phase Equilibr. 295 (2010) 98-103.

[20] Y. Guan, T.H. Lilley, T.E. Treffry, A new excluded volume theory and its application to the coexistence curves of aqueous polymer two-phase systems, Macromolecules 26 (1993) 3971-3979.

[21] G. Torres-Plasencia, E. Gutiérrez-Arnillas, F.J. Deive, M.A. Sanromán, A. Rodríguez, Triggering phase disengagement of 1-alkyl-3-methylimidazolium chloride ionic liquid by using inorganic and organic salts, J. Chem. Thermodynamics 88 (2015) 1-7. 
468 [22] H. Passos, A.R. Ferreira, A.F.M. Cláudio, J.A.P. Coutinho, M.G. Freire,

469

470

471

472

473

474

475

476

477

478

479

480

481

482

483

484

485

486

487

488

489

490

491

492

Characterization of Aqueous Biphasic Systems Composed of Ionic Liquids and a Citrate-based Biodegradable Salt, Biochem. Eng. J. 67 (2012) 68-76.

[23] H. Passos, M.P. Trindade, T.S.M. Vaz, L.P. da Costa, M.G. Freire, J.A.P. Coutinho, The Impact of Self-aggregation on the Extraction of Biomolecules in Ionic-liquid-based Aqueous Two-phase Systems, Sep. Purif. Technol. 108 (2013) 174-180.

[24] T.B.V. Dinis, H. Passos, D.L.D. Lima, V.I. Esteves, J.A.P. Coutinho, M.G. Freire, One-step Extraction and Concentration of Estrogens for an Adequate Monitoring of Wastewaters Using Ionic-Liquid-Based Aqueous Biphasic Systems, Green Chem. 17 (2015) 2570-2579.

[25] F.A. e Silva, T. Sintra, S.P.M. Ventura, J.A.P. Coutinho, Recovery of paracetamol from pharmaceutical wastes, Sep. Purif. Technol. 122 (2014) 315-322.

[26] T.E. Sintra, R. Cruz, S.P.M. Ventura, J.A.P. Coutinho, Phase diagrams of ionic liquids-based aqueous biphasic systems as a platform for extraction processes, J. Chem. Thermodyn. 77 (2014) 206-213.

[27] S.P.M. Ventura, S.G. Sousa, L.S. Serafim, A.S. Lima, M.G. Freire, J.A.P. Coutinho, Ionic Liquid Based Aqueous Biphasic Systems with Controlled pH: The Ionic Liquid Cation Effect, J. Chem. Eng. Data 56 (2011) 4253-4260.

[28] S.P.M. Ventura, S.G. Sousa, L.S. Serafim, A.S. Lima, M.G. Freire, J.A.P. Coutinho, Ionic Liquid Based Aqueous Biphasic Systems with Controlled pH: The Ionic Liquid Anion Effect, J. Chem. Eng. Data 57 (2012) 507-512.

[29] M. Taha, M.R. Almeida, F.A. e Silva, P. Domingues, S.P.M. Ventura, J.A.P. Coutinho, M.G. Freire, Novel Biocompatible and Self-buffering Ionic Liquids for Biopharmaceutical Applications, Chem. Eur. J. 21 (2015) 4781-4788.. 
493 [30] M. Taha, F.A. e Silva, M.V. Quental, S.P.M. Ventura, M.G Freire, J.A.P.

494 Coutinho, Good's buffers as a basis for developing self-buffering and

495 biocompatible ionic liquids for biological research, Green Chem. 16 (2014) 3149-

$496 \quad 3159$.

497 [31] J. Esteban, E. Fuente, A. Blanco, M. Ladero, F. Garcia-Ochoa, Phenomenological 498 kinetic model of the synthesis of glycerol carbonate assisted by focused beam reflectance measurements, Chem. Eng. J. 260 (2015) 434-443. 